\title{
Protecting genes from RNA silencing by destroying aberrant transcripts
}

\author{
ZHANG Heng $^{1 *} \&$ ZHU JianKang ${ }^{1,2}$ \\ ${ }^{1}$ Shanghai Center for Plant Stress Biology, Shanghai Institutes for Biological Sciences, Chinese Academy of Sciences, Shanghai 201602, \\ China; \\ ${ }^{2}$ Department of Horticulture and Landscape Architecture, Purdue University, West Lafayette, IN 47907, USA
}

Received May 6, 2015; accepted May 8, 2015; published online May 19, 2015

Citation: Zhang H, Zhu JK. Protecting genes from RNA silencing by destroying aberrant transcripts. Sci China Life Sci, 2015, 58: 613-615, doi: $10.1007 / \mathrm{s} 11427-015-4871-\mathrm{y}$

Gene expression is intensively regulated at the transcript level. It is the rate of transcription and the rate of degradation that together determine the steady-state level of a specific transcript. A whole-genome study indicates that the half-life of Arabidopsis transcripts varies from 0.2 to $>24 \mathrm{~h}$, indicating large variations in the turnover rate of specific transcripts, which is related to their biological functions [1]. While RNA polymerase II is responsible for mRNA production in all eukaryotic organisms, a large repertoire of ribonucleases are involved in mRNA decay [2]. Different components in the RNA degradation pathway usually target specific sets of transcripts. The specificity can be at least partly accounted for by the presence of RNA motifs within the 3' UTR regions and RNA binding proteins that recognize them. In Arabidopsis, mRNAs targeted for degradation are first deadenylated and subjected to digestion by at least two major classes of exonucleases [3]. The exosome performs $3^{\prime}-5^{\prime}$ degradation, whereas the XRN4 exonuclease acts on further decapped transcripts and digests them from the $5^{\prime}-$ end.

Both animals and plants utilize small RNAs for posttranscriptional gene silencing (PTGS) and/or transcriptional gene silencing (TGS). Evolutionarily, small RNA- mediated gene silencing is believed to function in defense against foreign nucleic acids, whether they are from viruses, transposons or transgenes. In Arabidopsis, small RNAs are usually 21-24 nucleotides in length. Different classes of small RNAs (sRNA) all require a double stranded RNA (dsRNA) precursor that is diced into small fragments by the RNase III-class DICER enzymes. The DICER protein functions like a ruler to determine the size of small RNA products. For example, siRNA generated by DCL2 are usually 22-nt long and siRNA by DCL4 are 21-nt long. When the guide strand of double stranded sRNAs are loaded into ARGONAUTE (AGO) proteins and form the RNA-induced silencing complex (RISC), the RISC complex binds to complementary transcripts, resulting in transcript cleavage or translational inhibition. The cleaved transcript can also be converted into dsRNAs by the RNA-dependent RNA polymerases (RDRs) and produce so-called secondary small interfering RNAs, which increases siRNA levels and ensures silencing.

The dsRNA precursor can be generated from different sources. MicroRNA (miRNA) genes produce transcripts that form hairpin-loop structures and are processed into mature miRNAs by DCL1. In other cases, aberrant transcripts are sensed by the cell and converted into dsRNAs by RDRs. For example, TRANS-ACTING SIRNA (TAS) genes are targets of miRNAs. The cleaved transcripts by the

*Corresponding author (email: zhangheng@sibs.ac.cn) 
miRNA-containing RISC complex are uncapped in the $5^{\prime}$ end and processed into 21-22 nt siRNAs by the combined action of RDR6 and DCL2/4. The resulting ta-siRNAs then regulate other genes in trans.

An interesting question is how the cell protects its own protein-coding transcripts from being targeted by the RNA silencing machinery. A recent study by Zhang et al. indicates that removal of aberrant transcripts by the RNA decay pathway is the key [4].

The authors started by screening for mutants that suppress the ethylene hyper-response phenotype of EIN3 overexpression lines. The result is the identification of several genes functioning in RNA degradation, including the 5'-3' exonuclease XRN4/EIN5 and subunits of the SKI complex that has RNA helicase activity and functions in feeding transcripts into the exosome for degradation. EIN5 was shown to suppress PTGS of specific transgenes and it was proposed that defects in $5^{\prime}-3^{\prime}$ RNA degradation lead to accumulation of aberrant transgene transcripts, which are targeted by the RNA silencing machinery [5]. Interestingly, whereas the ein 5 or the ski2 single mutant shows relatively normal phenotypes, the ein5-1 ski2-2 double mutant is embryonic lethal, indicating that EIN5 and SKI2 redundantly target some endogenous transcripts. By using the weak allele ski2-3, the ein5-1 ski2-3 mutant is viable but shows severe developmental defects. Surprisingly, the mutation in RDR6 almost completely restores normal phenotypes in ein5-1 ski2-3 mutants. Further genetic analyses indicated that the mutant phenotype of ein5 ski2 also depends on other known components of PTGS, including AGO1, SGS3 and DCL2/4. Thus disruption of both the $5^{\prime}$ and $3^{\prime}$ RNA decay function is devastating to plants. This might be because some highly expressed genes tend to generate aberrant RNAs such as abnormally processed RNAs, which are ordinarily destroyed by the RNA decay pathways. However, the aberrant RNAs would over-accumulate in the ein5 ski2 RNA decay mutants, and the accumulated aberrant RNAs may be channeled to the RDR6 protein, which initiates the RNA interfering process, leading to silencing of the transcripts.

Whole-genome mRNA and small RNA profiling provides direct support for the above model. Transcriptome analyses identified 596 differentially expressed genes in ein5 ski2 and $>80 \%$ of them were down-regulated. Very similar transcriptome profiles were observed between $r d r 6$ and ein5 ski2 rdr6, both of which show almost opposite pattern to that of the ein 5 ski2 mutant. In addition, more than 400 protein-coding genes that produce RDR6dependent sRNAs in the ein5 ski2 mutant were identified and named coding transcript-derived siRNAs (ct-siRNAs). As expected the ct-siRNA loci is over-represented in the down-regulated genes in the ein5-1 ski2-3 mutant, whose expression is presumably repressed by the ct-siRNAs. However, the up-regulated genes are also enriched for ct-siRNAs, indicating that either the siRNA does not have a silencing function or another antagonizing mechanism exists to protect the transcript from being destructed.

Considering the definition of ct-siRNAs, we may expect to identify more genes that produce them under different mutant backgrounds or environmental stresses. In the ein5 single mutant, though morphologically normal, accumulation of gene-derived siRNAs was reported for over one hundred loci [6]. The mRNA decapping enzymes, which function before XRN4 decay, also have a role in preventing PTGS of transgenes [7]. More importantly, other conditions that lead to accumulation of aberrant transcripts may also trigger the production of ct-siRNAs. Mutations defective in genes involved in RNA splicing and mRNA 3' end formation also enhanced PTGS at specific loci [8]. Recently it was proposed that splicing is likely an important feature by which the cell senses foreign/aberrant transcripts. The study in human pathogenic yeast Cryptococcus neoformans shows that transposons tend to have sub-optimal splicing sites that may be sensed by spliceosomes and enhance siRNA biogenesis [9]. Indeed Arabidopsis intron-less genes have higher exon siRNA density, and introducing an intron into a GFP transgene suppresses spurious transgene silencing in plants [10]. Thus cells probably utilized every feature of normal mRNAs to recognize aberrant/foreign ones. A proper function of the RNA decay and RNA processing pathways is important to protect normal transcripts from being targeted for gene silencing.

1 Narsai R, Howell KA, Millar AH, O'Toole N, Small I, Whelan J. Genome-wide analysis of mRNA decay rates and their determinants in Arabidopsis thaliana. Plant Cell, 2007, 19: 3418-3436

2 Stoecklin G, Muhlemann O. RNA decay mechanisms: specificity through diversity. Biochim Biophys Acta, 2013, 1829: 487-490

3 Belostotsky DA, Sieburth LE. Kill the messenger: mRNA decay and plant development. Curr Opin Plant Biol, 2009, 12: 96-102

4 Zhang XY, Zhu Y, Liu XD, Hong XY, Xu Y, Zhu P, Shen Y, Wu HH, Ji YS, Wen X, Zhang C, Zhao Q, Wang YC, Lu J, Guo HW. Suppression of endogenous gene silencing by bidirectional cytoplasmic RNA decay in Arabidopsis. Science, 2015, 348: 120-123

5 Gazzani S, Lawrenson T, Woodward C, Headon D, Sablowski R. A link between mRNA turnover and RNA interference in Arabidopsis. Science, 2004, 306: 1046-1048

6 Gregory BD, O'Malley RC, Lister R, Urich MA, Tonti-Filippini J, Chen H, Millar AH, Ecker JR. A link between RNA metabolism and silencing affecting Arabidopsis development. Dev Cell, 2008, 14: 854-866

7 Thran M, Link K, Sonnewald U. The Arabidopsis DCP2 gene is required for proper mRNA turnover and prevents transgene silencing 
in Arabidopsis. Plant J, 2012, 72: 368-377

8 Herr AJ, Molnar A, Jones A, Baulcombe DC. Defective RNA processing enhances RNA silencing and influences flowering of Arabidopsis. Proc Natl Acad Sci USA, 2006, 103: 14994-15001

9 Dumesic PA, Natarajan P, Chen CB, Drinnenberg IA, Schiller BJ,
Thompson J, Moresco JJ, Yates JR, Bartel DP, Madhani HD. Stalled spliceosomes are a signal for RNAi-mediated genome defense. Cell, 2013, 152: 957-968

10 Christie M, Croft LJ, Carroll BJ. Intron splicing suppresses RNA silencing in Arabidopsis. Plant J, 2011, 68: 159-167

Open Access This article is distributed under the terms of the Creative Commons Attribution License which permits any use, distribution, and reproduction in any medium, provided the original author(s) and source are credited. 